\title{
Atrial fibrillation type and renal dysfunction as important predictors of left atrial thrombus
}

\author{
Agnieszka Kapłon-Cieślicka, ${ }^{1}$ Monika Budnik, ${ }^{1}$ Monika Gawałko, ${ }^{\oplus 1}$ Michał Peller, ${ }^{1}$ \\ Iwona Gorczyca, ${ }^{2}$ Anna Michalska, ${ }^{2}$ Aldona Babiarz, ${ }^{1}$ Aleksandra Bodys, ${ }^{1}$ \\ Robert Uliński, ${ }^{1}$ Maciej Żochowski, ${ }^{1}$ Piotr Scisło, Janusz Kochanowski, ${ }^{1}$ \\ Krzysztof J Filipiak, ${ }^{1}$ Grzegorz Opolski ${ }^{1}$
}

- Additional material is published online only. To view please visit the journal online (http://dx.doi.org/10.1136/ bmjres-2018-314492)

${ }^{1} 1$ st Chair and Department of Cardiology, Medical University of Warsaw, Warsaw, Poland

${ }^{2} 1$ st Department of Cardiology and Electrotherapy,

Świętokrzyskie Cardiology

Centre, Kielce, Poland

\section{Correspondence to}

Dr Monika Gawałko;

mongawalko@gmail.com

Received 16 November 2018

Revised 1 April 2019

Accepted 8 April 2019

Published Online First

30 April 2019

\section{Linked}

- http://dx.doi.org/10.1136/ heartjnl-2019-315212

Check for updates

(C) Author(s) (or their employer(s)) 2019. No commercial re-use. See rights and permissions. Published by BMJ.

To cite: Kapłon-Cieślicka A, Budnik M, Gawałko M, et al. Heart 2019;105:1310-1315.

\section{ABSTRACT}

Objective We aimed to identify predictors of left atrial appendage (LAA) thrombus in patients with atrial fibrillation (AF) and to enhance the prognostic value of the CHA DS -VASC score.

Methods Derivation cohort included 1033 consecutive AF patients referred for catheter ablation or direct current cardioversion, in whom transoesophageal echocardiography (TOE) was performed prior to the procedure. Logistic regression analysis was used to identify predictors of LAA thrombus on TOE. Receiver operating characteristic (ROC) curves were constructed to compare the newly developed score with the $\mathrm{CHA}_{2} \mathrm{DS}_{2}$ and $\mathrm{CHA}_{2} \mathrm{DS}_{2}$-VASc scores in the derivation and the validation $(n=320)$ cohort.

Results On TOE, LAA thrombus was present in $59(5.7 \%)$ patients in the derivation cohort. Aside from variables encompassed by the $\mathrm{CHA}_{2} \mathrm{DS}_{2}$-VASC score, LAA thrombus predictors included AF type (persistent/'permanent' vs paroxysmal) and renal dysfunction. These predictors were incorporated into the $\mathrm{CHA}_{2} \mathrm{DS}_{2}$-VASC score. In ROC analysis, area under the curve (AUC) for the new score ( $\mathrm{CHA}_{2} \mathrm{DS}_{2}$-VASC-RAF score) was significantly higher (0.81) than those for the $\mathrm{CHA}_{2} \mathrm{DS}_{2}$ and $\mathrm{CHA}_{2} \mathrm{DS}$, -VASc scores (0.71 and 0.70, respectively). In the validation cohort, the $\mathrm{CHA}_{2} \mathrm{DS}_{2}$-VASCRAF score also performed significantly better (AUC of 0.88 ) than the $\mathrm{CHA}_{2} \mathrm{DS}_{2}$ and $\mathrm{CHA}_{2} \mathrm{DS} \mathrm{S}_{2}$-VASC scores (AUC of 0.63 and 0.60 , respectively).

Conclusion In real-world AF patients with majority on oral anticoagulation, LAA thrombus was found in approximately $6 \%$. Two variables not included in the $\mathrm{CHA}_{2} \mathrm{DS}_{2}$-VASc score (AF type and renal dysfunction) proved strong, independent predictors of LAA thrombus and might improve thromboembolic risk stratification.

\section{INTRODUCTION}

Atrial fibrillation (AF) is a well-established risk factor for thromboembolic complications, including ischaemic stroke, by promoting left atrial appendage (LAA) clot formation. ${ }^{1}$ The risk of stroke in patients with $\mathrm{AF}$ is amplified by a convergence of other factors, that is, age, co-existing clinical conditions and cardiac structure. Currently, the $\mathrm{CHA}_{2} \mathrm{DS}_{2}$ VASc score is recommended for thromboembolic risk stratification and establishing indications for oral anticoagulation in patients with non-valvular $\mathrm{AF}^{2}{ }^{2}$ However, the $\mathrm{CHA}_{2} \mathrm{DS}_{2}$-VASc score might not include all relevant factors that substantially increase the risk of LAA thrombus formation..$^{3-6}$ Furthermore, even anticoagulated patients with AF are still at residual risk of LAA clot formation and might experience thromboembolic events.

The aim of the study was to assess the prevalence of LAA thrombus in a real-world population of treated $\mathrm{AF}$ patients, to identify clinical predictors of LAA thrombus in those patients and to enhance the prognostic value of the $\mathrm{CHA}_{2} \mathrm{DS}_{2}$-VASc score by including additional risk factors for LAA clot formation.

\section{METHODS}

\section{Patient population}

The derivation cohort included consecutive AF patients referred to an academic cardiology department between January 2012 and August 2016 for catheter ablation (pulmonary vein isolation) or direct current cardioversion for AF. All those patients had transoesophageal echocardiography (TOE) performed prior to the procedure. The validation cohort consisted of consecutive AF patients admitted to a different cardiology department in a district hospital between January 2013 and December 2017, who underwent TOE before catheter ablation (pulmonary vein isolation) or direct current cardioversion of AF.

All clinical, laboratory and echocardiographic (including TOE) data were obtained retrospectively from medical records. Patients were included in the study regardless of the presence or type of anticoagulation prior to TOE.

Research protocol and retrospective review of medical records were approved by the ethics committee of the Medical University of Warsaw. As this was an observational, retrospective study, the ethics committee waived the requirement of obtaining informed consent from the patients.

Patients were divided into three groups based on AF type: paroxysmal, persistent and 'permanent' $\mathrm{AF}$, based on a careful and thorough analysis of all the available medical documentation, including current and previous medical records, electrocardiograms and, in some patients, Holter monitoring (if available). Patients were classified as having 'permanent' AF after unsuccessful cardioversion during index hospitalisation or if they had been previously diagnosed with permanent $\mathrm{AF}$, and the 
diagnosis was subsequently changed to long-standing persistent AF before cardioversion or ablation; these patients were classified as 'permanent' AF to distinguish them from persistent AF patients with presumably lower AF burden. Data on exact AF duration were not available for all patients, and thus, a reliable diagnosis of long-standing persistent AF could not have been made. Similarly, no data on exact AF burden from Holter monitoring were available for all patients.

Glomerular filtration rate (GFR) was calculated using Modification of Diet in Renal Disease Study equation.

\section{Transoesophageal echocardiography}

In the academic department (derivation cohort), all patients scheduled for direct current cardioversion of $\mathrm{AF}$ or for catheter ablation of AF, irrespective of the presence or type of anticoagulation, have TOE performed routinely prior to the procedure, with the exception of patients in whom direct current cardioversion is conducted for emergency indications. ${ }^{7}$ In the district hospital department (validation cohort), TOE studies before cardioversion are performed at the discretion of the attending physician, that is, if there is any doubt regarding the efficacy of oral anticoagulation or patient's compliance. In both departments, TOE examinations are usually conducted directly or within a few hours prior to the scheduled procedure (at most within 48 hours before the procedure). All TOE studies in the derivation cohort were performed by certified echocardiographers (second-degree accreditation in echocardiography of the Section of Echocardiography of the Polish Cardiac Society (PCS)), using EPIQ 7 Ultrasound Machine (Philips Medical Systems, Andover, Massachusetts, USA) or iE33 Ultrasound Machine (Philips Medical Systems) with an X72t TOE ultrasound transducer (Philips Medical Systems), in the department's echocardiography laboratory (certified with grade $\mathrm{C}$ accreditation of the Section of Echocardiography of the PCS). When LAA thrombus was suspected, the study was evaluated by two echocardiographers, and in doubtful cases, by a third echocardiographer, to establish a unanimous and most reliable diagnosis and to enable safe referral for ablation or cardioversion. All TOE examinations were recorded and stored, and available for re-evaluation, if needed. All TOE studies in the validation cohort were performed by experienced echocardiographers using iE33 Ultrasound Machine (Philips Medical Systems) with an X72t TOE ultrasound transducer (Philips Medical Systems) in the department's echocardiography laboratory (certified with accreditation of the Section of Echocardiography of the PCS). Written informed consent for TOE was obtained from all patients.

\section{Study endpoint}

The primary endpoint was the presence of left atrial thrombus on TOE.

\section{Statistical analysis}

Continuous and ordinal data were presented as median and IQR. Group comparisons were conducted using the Fisher exact test for qualitative variables, the t-test for quantitative, normally distributed variables and the Mann-Whitney $\mathrm{U}$ test for quantitative, non-normally distributed variables (normality of distribution was checked with the Shapiro-Wilk test). The Kruskal-Wallis test was used to compare patients with paroxysmal, persistent and 'permanent' AF. To determine predictors of LAA thrombus on TOE, univariate and multivariate logistic regression analyses were performed in the derivation cohort. Additionally, the Brier score was calculated for newly identified predictors of LAA thrombus. Online supplementary table S1 presents variables included in univariate logistic regression analyses. Multivariate logistic regression model included all variables found to be predictors of LAA thrombus in univariate analyses, maintaining an adequate event per predictor variable value. ${ }^{8} \mathrm{~A}$ second multivariate model was derived, including the $\mathrm{CHA}_{2} \mathrm{DS}_{2}-\mathrm{VASc}$ score, to enable adequate point attribution. All variables included in these models were qualitative variables (to enable point attribution), that is, quantitative variables (such as GFR) were converted into qualitative variables, using the Youden index to estimate the optimal cut-off for a given variable. Receiver operating characteristic (ROC) curves were constructed and area under the curve (AUC) was calculated to compare the sensitivity and specificity of different risk scores in identification of patients with LAA thrombus in both patient cohorts. Pairwise comparison of ROC curves was performed using Mann-Whitney U statistics. Additionally, net reclassification improvement for the newly derived model was calculated. For all tests, a p value of less than 0.05 was considered significant. All tests were two-tailed. All calculations were performed using the SASV.9.2.

\section{RESULTS}

\section{Derivation cohort characteristics}

The derivation cohort included 1033 patients. Median age was 60 years (IQR: 53-66 years), median $\mathrm{CHA}_{2} \mathrm{DS}_{2}$-VASc score was 2 (IQR: 1-3) and 34\% were female. AF was classified as paroxysmal in $61 \%$, persistent in $33 \%$ and 'permanent' in $6 \%$ of patients. Forty-five percent of patients were treated with vitamin $\mathrm{K}$ antagonists (VKA), 44\% with non-VKA oral anticoagulants (including $23 \%$ on rivaroxaban, $20 \%$ on dabigatran and one patient on apixaban) and $11 \%$ did not receive any chronic oral anticoagulation.

On TOE, left atrial thrombi were detected in $59(5.7 \%$; $95 \%$ CI : $4.4 \%$ to $7.3 \%$ ) patients of the derivation cohort. All of those thrombi were found in LAA. Online supplementary table S2 presents the characteristics of patients with and without LAA thrombi on TOE.

\section{Predictors of left atrial thrombus in the derivation cohort}

Online supplementary table S1 shows variables included in univariate logistic regression analyses of LAA thrombus predictors. International normalised ratio (INR) and activated partial thromboplastin time (APTT) were not included in the analyses, as patients were enrolled in the study irrespective of the type of oral anticoagulation and of prior bridging therapy with heparin (58 patients received bridging therapy with unfractionated heparin or low-molecular-weight heparin) and as VKA were temporarily stopped before the procedure in some (but not all) ablation patients. Lack of oral anticoagulation, type of oral anticoagulation, as well as receiving bridging therapy prior to the procedure were not predictors of LAA thrombi on TOE in univariate analyses.

Variables found to be significant predictors of LAA thrombus in univariate analyses included factors encompassed by the $\mathrm{CHA}_{2} \mathrm{DS}_{2}$-VASc score, such as older age, diabetes, heart failure and prior stroke, transient ischaemic attack or peripheral embolism, as well as factors not included in the $\mathrm{CHA}_{2} \mathrm{DS}_{2}$-VASc score, that is, persistent and 'permanent' AF (vs paroxysmal AF), chronic respiratory disease and lower GFR. All these factors were consequently included in the multivariate model (table 1). All quantitative variables were expressed as qualitative data, that is, age was expressed as age $\geq 75$ years and age 65-74 years (as in the $\mathrm{CHA}_{2} \mathrm{DS}_{2}$-VASc score), and for GFR an optimal cut-off 
Table 1 Logistic regression analyses of predictors of left atrial thrombus in the derivation cohort

\begin{tabular}{|c|c|c|c|c|}
\hline \multirow[b]{2}{*}{ Variable } & \multirow{2}{*}{$\begin{array}{l}\text { Univariate } \\
\text { analyses } \\
P \text { value }\end{array}$} & \multicolumn{3}{|c|}{ Multivariate analysis } \\
\hline & & OR & $95 \% \mathrm{Cl}$ & $P$ value \\
\hline Age $\geq 75$ years & $<0.0001$ & 2.71 & 1.18 to 6.19 & 0.018 \\
\hline Age $65-74$ years & 0.01 & 1.60 & 0.80 to 3.21 & 0.18 \\
\hline Diabetes & 0.0015 & 1.25 & 0.64 to 2.46 & 0.51 \\
\hline Heart failure & $<0.0001$ & 2.74 & 1.45 to 5.19 & 0.002 \\
\hline $\begin{array}{l}\text { Prior stroke/ } \\
\text { TIA/peripheral embolism }\end{array}$ & 0.01 & 1.82 & 0.78 to 4.27 & 0.17 \\
\hline Respiratory disease & 0.02 & 1.53 & 0.58 to 4.03 & 0.39 \\
\hline Persistent AF & $<0.0001$ & 5.08 & 2.41 to 10.70 & $<0.0001$ \\
\hline Permanent AF & $<0.0001$ & 9.78 & 3.85 to 24.85 & $<0.0001$ \\
\hline $\begin{array}{l}\mathrm{GFR}<56 \mathrm{~mL} / \\
\mathrm{min} / 1.73 \mathrm{~m}^{2}\end{array}$ & $<0.0001$ & 2.27 & 1.24 to 4.16 & 0.008 \\
\hline
\end{tabular}

$\mathrm{AF}$, atrial fibrillation; GFR, glomerular filtration rate; TIA, transient ischaemic attack. Bold values indicate statistically significant data ie. with $p$ value $<0.05$.

was established based on the Youden index, which was GFR of $<56 \mathrm{~mL} / \mathrm{min} / 1.73 \mathrm{~m}^{2}$. In multivariate analysis, along with a few components of the $\mathrm{CHA}_{2} \mathrm{DS}_{2}$-VASc score, AF type (persistent and 'permanent' $\mathrm{AF}$ ), as well as renal dysfunction (defined as GFR $<56 \mathrm{~mL} / \mathrm{min} / 1.73 \mathrm{~m}^{2}$ ) remained independent predictors of LAA thrombus.

The Brier score for AF type was 0.05 , for renal dysfunction- 0.05 and for the combination of both factors- 0.048 . AUC for AF type and renal dysfunction were 0.75 and 0.65 , respectively.

\section{Subanalyses in patients with left atrial thrombus in relation to AF type and renal dysfunction}

Online supplementary table S3 presents characteristics of patients with LAA thrombus depending on AF type. Online supplementary table $\mathrm{S} 4$ shows characteristics of patients with LAA thrombus depending on the presence of renal dysfunction (defined as GFR $<56 \mathrm{~mL} / \mathrm{min} / 1.73 \mathrm{~m}^{2}$ ).

\section{Antithrombotic treatment in patients with renal dysfunction}

Antithrombotic treatment of patients with renal dysfunction is shown in online supplementary table S5. The derivation cohort included seven patients with GFR $<15 \mathrm{~mL} / \mathrm{min} / 1.73 \mathrm{~m}^{2}$ and six patients with GFR between 15 and $30 \mathrm{~mL} / \mathrm{min} / 1.73 \mathrm{~m}^{2}$.

\section{Development of a new score for the prediction of left atrial thrombus}

In univariate analyses, both the CHADS 2 and the $\mathrm{CHA}_{2} \mathrm{DS}_{2}$ VASc scores predicted LAA thrombus on TOE in the derivation cohort, with OR of 2.27 (for 1 point), $95 \%$ CI of 1.73 to 2.98 and $\mathrm{p}<0.0001$ for the CHADS, score and OR of 1.60 (for 1 point), $95 \% \mathrm{CI}$ of 1.36 to 1.88 and $\mathrm{p}<0.0001$ for the $\mathrm{CHA}_{2} \mathrm{DS}_{2}-$ VASc score.

In an attempt to derive a new, $\mathrm{CHA}_{2} \mathrm{DS}_{2}$-VASc-based risk score, we developed a second multivariate model, including the $\mathrm{CHA}_{2} \mathrm{DS}_{2}$-VASc score, together with variables found to independently predict LAA thrombus in our first (table 1) multivariate analysis, that is, $\mathrm{AF}$ type and renal dysfunction $\left(\mathrm{GFR}<56 \mathrm{~mL} / \mathrm{min} / 1.73 \mathrm{~m}^{2}\right)$. This second multivariate model is presented in table 2 . In this model, all included components proved strong, independent predictors of LAA thrombus. In order to establish scoring for the new components of the developed risk model, we compared ORs of each new variable (ie,
Table 2 Predictors of left atrial thrombus in the derivation cohort: multivariate logistic regression model, including $\mathrm{CHA}_{2} \mathrm{DS}_{2}$-VASc score

\begin{tabular}{lclc}
\hline & \multicolumn{3}{c}{ Multivariate analysis } \\
\cline { 2 - 4 } Variable & OR & $95 \% \mathrm{Cl}$ & P value \\
\hline CHA $_{2} \mathrm{DS}_{2}$-VASc score (per 1 point) & 1.36 & 1.14 to 1.62 & $\mathbf{0 . 0 0 0 8}$ \\
\hline Persistent AF & 5.76 & 2.77 to 11.95 & $<0.0001$ \\
Permanent AF & 13.02 & 5.30 to 32.00 & $<0.0001$ \\
GFR $<56 \mathrm{~mL} / \mathrm{min} / 1.73 \mathrm{~m}^{2}$ & 2.41 & 1.33 to 4.37 & $\mathbf{0 . 0 0 4}$ \\
\hline AF,
\end{tabular}

$\mathrm{AF}$, atrial fibrillation; $\mathrm{GFR}$, glomerular filtration rate. Bold values indicate statistically significant data ie. with $\mathrm{p}$ value $<0.05$.

persistent or 'permanent' AF and renal dysfunction) with the OR of 1 point in the CHA,DS -VASc score. Thus, persistent AF was attributed 4 points (as 5.76 divided by 1.36 equals 4.24 ), 'permanent' AF-10 points (as 13.02 divided by 1.36 equals 9.57 ) and renal dysfunction-2 points (as 2.41 divided by 1.36 equals 1.77). This new, extended version of the $\mathrm{CHA}_{2} \mathrm{DS}_{2}$-VASc score was called $\mathrm{CHA}_{2} \mathrm{DS}_{2}$-VASc-RAF score ( $\mathrm{R}$ for Renal dysfunction and $\mathrm{AF}$ for $\mathrm{AF}$ type) and is presented in table 3.

Next, we plotted ROC curves for the $\mathrm{CHADS}_{2}$, the $\mathrm{CHA}_{2} \mathrm{DS}_{2}$ VASc and the newly derived $\mathrm{CHA}_{2} \mathrm{DS}_{2}$-VASc-RAF score, as shown in figure 1. Pairwise comparison of the ROC curves showed that the differences between the $\mathrm{CHADS}_{2}$ and the $\mathrm{CHA}_{2} \mathrm{DS}_{2}-$ VASc-RAF score as well as between the CHA $\mathrm{DS}_{2}$-VASc and the $\mathrm{CHA}_{2} \mathrm{DS}_{2}$-VASc-RAF score were highly significant (with both $\mathrm{p}$ values of 0.0003 ).

Based on the Youden index, the optimal cut-off for the $\mathrm{CHA}_{2} \mathrm{DS}_{2}$-VASc-RAF score to predict LAA thrombus in the derivation cohort might be set at 5 points for men and at 6 points for women (with sensitivity of 0.83 and specificity of $0.74)$. In the derivation cohort, sensitivity and specificity of the $\mathrm{CHA}_{2} \mathrm{DS}_{2}$-VASc score in identifying patients with LAA thrombus were 0.64 and 0.81 , respectively (for the cut-off of 2 points in men and 3 points in women-as thresholds for class I indication for oral anticoagulation according to the current European Society of Cardiology (ESC) guidelines ${ }^{2}$ ). Including the above-mentioned cut-off points for the CHA, $\mathrm{DS}_{2}$-VASc and the $\mathrm{CHA}_{2} \mathrm{DS}_{2}$-VASc-RAF scores, net reclassification improvement in the derivation cohort was 0.138 . Table 4 illustrates risk reclassification in the derivation cohort.

Table 3 Proposal of a new, $\mathrm{CHA}_{2}$ DS - -VASc score-based risk stratification model: the $\mathrm{CHA}_{2} \mathrm{DS}_{2}$-VASc-RAF score

\begin{tabular}{lllc}
\hline & Condition & Definition & Points \\
\hline $\mathrm{C}$ & Congestive heart failure & As in the & 1 \\
$\mathrm{H}$ & Hypertension & CHA $_{2} \mathrm{DS}_{2}$-VASc score & 1 \\
$\mathrm{~A}_{2}$ & Age $\geq 75$ years & & 2 \\
$\mathrm{D}$ & Diabetes & & 1 \\
$\mathrm{~S}_{2}$ & Stroke or TIA or & & 2 \\
& thromboembolism & & 1 \\
$\mathrm{~V}$ & Vascular disease & & 1 \\
$\mathrm{~A}$ & Age 65-74years & & 1 \\
$\mathrm{Sc}$ & Sex category (female sex) & GFR $<56 \mathrm{~mL}$ & 2 \\
$\mathrm{R}$ & Renal dysfunction & min/1.73 m & \\
& & Persistent AF & 4 \\
$\mathrm{AF}$ & AF type & Permanent or long- & 10 \\
& & standing persistent & \\
& & AF & \\
\hline
\end{tabular}

AF, atrial fibrillation; GFR, glomerular filtration rate; TIA, transient ischaemic attack. 
DERIVATION COHORT

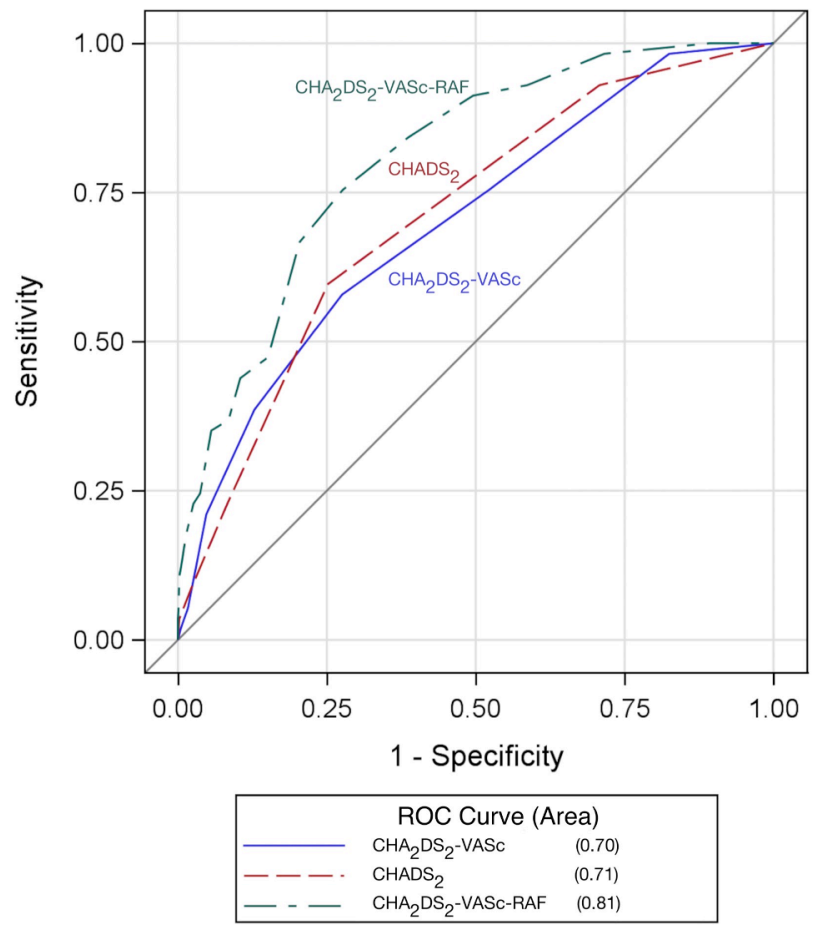

VALIDATION COHORT



Figure 1 Receiver operating characteristic (ROC) curves and area under the curve for the $\mathrm{CHADS}_{2}$, the $\mathrm{CHA}_{2} \mathrm{DS}_{2}$-VASC and the new CHA 2 DS - -VASCRAF score in the derivation and validation cohort.

\section{Validation of the new score}

The validation cohort included 320 patients. On TOE, left atrial thrombi were detected in $23(7.2 \%$; 95\% CI: $4.6 \%$ to $10.6 \%)$ of these patients. Characteristics of the validation cohort are presented in online supplementary table S6. ROC curves for the $\mathrm{CHADS}_{2}$, the $\mathrm{CHA}_{2} \mathrm{DS}_{2}$-VASc and the $\mathrm{CHA}_{2} \mathrm{DS}_{2}$-VASc-RAF score are shown in figure 1 . Pairwise comparison of the ROC curves showed that the differences between the $\mathrm{CHADS}_{2}$ and the $\mathrm{CHA}_{2} \mathrm{DS}_{2}$-VASc-RAF score as well as between the $\mathrm{CHA}_{2} \mathrm{DS}_{2}-$ VASc and the $\mathrm{CHA}_{2} \mathrm{DS}_{2}$-VASc-RAF score were highly significant (with $\mathrm{p}$ values of 0.0001 and $<0.0001$, respectively).

\section{DISCUSSION}

Although the current ESC guidelines do not list AF type or AF burden among factors affecting the probability of LAA thrombus formation, a few studies have shown that persistent or permanent AF carries a higher risk of stroke than paroxysmal AF. ${ }^{5910}$ A recent meta-analysis, including 99996 patients from

Table 4 Risk reclassification in the derivation cohort

\begin{tabular}{llll}
\hline & \multicolumn{3}{l}{$\mathrm{CHA}_{2} \mathrm{DS}_{2}$-VASC } \\
\cline { 3 - 3 } & & High risk & Low risk \\
\hline CHA $_{2}$ DS $_{2}$-VASC-RAF & High risk & Thrombus: 35 & Thrombus: 14 \\
& & No thrombus: 198 & No thrombus: 124 \\
& \multirow{2}{*}{ Low risk } & Thrombus: 3 & Thrombus: 7 \\
& & No thrombus: 191 & No thrombus: 452 \\
\hline
\end{tabular}

Risk categories

$\mathrm{CHA}_{2} \mathrm{DS}_{2}$-VASc score: high risk: $\geq 2$ points in men, $\geq 3$ points in women; low risk: 0-1 points in men, 0-2 points in women.

CHA ${ }_{2} \mathrm{DS}_{2}$-VASc-RAF score: high risk: $\geq 5$ points in men, $\geq 6$ points in women; low risk: $0-4$ points in men, $0-5$ pointsin women.
12 studies, revealed that in non-paroxysmal AF, the risk for thromboembolism is increased by 38\% compared with paroxysmal AF. ${ }^{11}$ Furthermore, in patients with paroxysmal AF, higher AF burden may be related to increased stroke risk. ${ }^{12}$ In our study, non-paroxysmal AF proved a strong, independent predictor of LAA thrombus on TOE.

The second important predictor of LAA thrombus, not included in the $\mathrm{CHA}_{2} \mathrm{DS}_{2}$-VASc score, was reduced GFR. Renal impairment has been previously shown to predict thromboembolism in AF. ${ }^{613-16}$ In both the ATRIA study and the $\mathrm{R}_{2} \mathrm{CHADS}_{2}$ score, renal dysfunction improved thromboembolic risk stratification. ${ }^{14} 17$ On the contrary, few smaller studies reported that inclusion of renal function has shown only modest improvement in the predictive value of the $\mathrm{CHADS}_{2}$ and the $\mathrm{CHA}_{2} \mathrm{DS}_{2}$-VASc scores, possibly because kidney disease is strongly associated with other components of these risk models (such as heart failure, hypertension or diabetes). ${ }^{18-21}$ Importantly, in our study, antithrombotic therapy in the subgroup with renal dysfunction was comparable with the treatment in the whole derivation cohort. Analogous to the whole group, there was no association between the type of antithrombotic treatment and the presence of LAA thrombus in patients with renal dysfunction. Still, it needs to be emphasised that our study included few patients with advanced kidney disease.

In our study, relative risk attributable to 1 point in the $\mathrm{CHA}_{2} \mathrm{DS}_{2}$-VASc score, as well as c-statistics of the $\mathrm{CHADS}_{2}$ and the $\mathrm{CHA}_{2} \mathrm{DS}_{2}$-VASc scores for the prediction of LAA thrombus, was consistent with those observed in previous studies. ${ }^{112} 1522-25$ The $\mathrm{CHA}_{2} \mathrm{DS}_{2}$-VASc-RAF score demonstrated good sensitivity and specificity in discriminating patients with LAA thrombus (AUC of 0.81-0.88), while the CHADS, and the CHA, DS 2 -VASc scores showed poor-to-fair sensitivity and specificity in both our 
cohorts (AUC of $0.60-0.71$ ) as well as in the previous studies (AUC ranging between 0.55 and 0.7 ). ${ }^{122-25}$ It may be argued that the scoring assigned for persistent and long-standing persistent/ permanent $\mathrm{AF}$ in the $\mathrm{CHA}_{2} \mathrm{DS}_{2}$-VASc-RAF score seems too high-especially given the wide confidence intervals in multivariate analyses (tables 1 and 2) - and might require recalibration in future cohorts. Nevertheless, even if the proposed scoring is not yet appropriate for routine clinical use, we feel that patients with non-paroxysmal AF and a $\mathrm{CHA}_{2} \mathrm{DS}_{2}$-VASc score of 0 or 1 point should not automatically be attributed low thromboembolic risk and disqualified from anticoagulant treatment.

\section{Limitations of the study}

The main limitation of our study is that its primary endpoint was the presence of left atrial thrombus on TOE (which is a surrogate endpoint) and not ischaemic stroke. However, LAA thrombus formation is considered the primary mechanism responsible for thromboembolic events in patients with AF; thus, the design of our study seems appropriate to address thromboembolic risk stratification in $\mathrm{AF}^{26-30}$

Second, due to lack of sufficient data we could not discriminate between persistent and long-standing persistent AF and had no information on the exact AF burden, which would seem relevant, given the results of our analysis. Distinguishing patients with 'permanent' AF from patients with persistent AF in our analysis was an attempt to discriminate between patients with presumably higher and lower AF burden.

Third, our real-life study group included patients irrespective of the presence or type of anticoagulation. Therefore, the derivation cohort was not homogenous, including patients with and without oral anticoagulation, with different anticoagulant regimens and with or without bridging therapy. Obviously, this made it impossible to include INR or APTT as variables in the logistic regression analysis of predictors of LAA thrombus. Only $11 \%$ of patients received no oral anticoagulation prior to TOE. Thus, the results of our analysis describe rather the residual risk of thrombus formation despite oral anticoagulation.

In our study, we did not assess the impact of some TOE-derived parameters (such as LAA emptying velocity or LAA morphology) on the risk of LAA thrombus formation. However, we aimed to determine clinical risk factors that would allow evaluation of pre-TOE probability of LAA thrombus, enhance the predictive value of the $\mathrm{CHA}_{2} \mathrm{DS}_{2}$-VASc score and be easy to obtain in every-day practice.

Finally, data in our study were retrieved retrospectively, and thus, data on some variables were not available for all patients, as indicated in tables. Importantly, data on left atrial size were available only for $40 \%$ of patients from the derivation cohort, and therefore, we could not include this variable in the logistic regression analysis of LAA thrombus predictors. Moreover, left atrial size was assessed only by its antero-posterior diameter and not by left atrial volume index, as recommended by the current guidelines. Still, we feel that the design of our study meets the requirements of a prospective observation, as we enrolled all consecutive patients with AF undergoing TOE.

\section{CONCLUSION}

In a real-world population of patients with AF referred for cardioversion or catheter ablation, with majority on oral anticoagulants, LAA thrombus was found in approximately $6 \%$. Two variables not included in the $\mathrm{CHA}_{2} \mathrm{DS}_{2}$-VASc score (AF type and renal dysfunction) proved strong, independent predictors of LAA thrombus on TOE and thereby might improve thromboembolic risk stratification. Nevertheless, the proposed $\mathrm{CHA}_{2} \mathrm{DS}_{2}$-VASc-RAF score requires validation in further studies.

\section{Key messages}

What is already known on this subject?

- The CHA $\mathrm{DS}_{2}$-VASc score is recommended for thromboembolic risk stratification and establishing indications for chronic oral anticoagulation in patients with non-valvular atrial fibrillation (AF).

\section{What might this study add?}

- In a real-world population of patients with $A F$, with majority on oral anticoagulation, left atrial appendage (LAA) thrombus was found in approximately $6 \%$. Two variables not included in the $\mathrm{CHA}_{2} \mathrm{DS}$-VASc score (AF type and renal dysfunction) proved strong, independent predictors of LAA thrombus. Incorporating these two variables into the $\mathrm{CHA}_{2} \mathrm{DS}_{2}-\mathrm{VASC}$ score significantly improved its prognostic value.

How might this impact on clinical practice?

- Our results suggest that patients with non-paroxysmal $\mathrm{AF}$ and a $\mathrm{CHA}_{2} \mathrm{DS}_{2}$-VASc score of 0 or 1 point should not automatically be disqualified from anticoagulant treatment. Including renal dysfunction and AF type in the $\mathrm{CHA}_{2} \mathrm{DS}_{2}-\mathrm{VASC}$ score might improve thromboembolic risk stratification in $\mathrm{AF}$.

Contributors $\mathrm{AK}-\mathrm{C}$ and $\mathrm{MB}$ were responsible for the concept and design of the study. MG, IG, AM, ABa, ABo, RU and $M Z$ were involved in data collection. AK-C, $M B, P S$ and JK performed echocardiographic evaluation. MP performed statistical calculations. AK-C and MP conducted data analysis and interpretation. AK-C and MG wrote the manuscript. All authors revised the manuscript and approved its final version.

Funding The authors have not declared a specific grant for this research from any funding agency in the public, commercial or not-for-profit sectors.

Competing interests None declared.

Patient consent for publication Obtained.

Provenance and peer review Not commissioned; externally peer reviewed.

\section{REFERENCES}

1. Lim HS, Willoughby SR, Schultz C, et al. Effect of atrial fibrillation on atrial thrombogenesis in humans: impact of rate and rhythm. J Am Coll Cardiol 2013;61:852-60

2. Kirchhof $P$, Benussi $S$, Kotecha D, et al. ESC Guidelines for the management of atrial fibrillation developed in collaboration with EACTS. Eur Heart J 2016;2016:2893-962.

3. Akoum N. New perspectives on atrial fibrillation and stroke. Heart 2016;102:1788-92.

4. Chao TF, Tsao HM, Ambrose K, et al. Renal dysfunction and the risk of thromboembolic events in patients with atrial fibrillation after catheter ablation-the potential role beyond the $\mathrm{CHA}_{2} \mathrm{DS}_{2}$-VASc score. Heart Rhythm 2012;9:1755-60.

5. Steinberg BA, Hellkamp AS, Lokhnygina Y, et al. Higher risk of death and stroke in patients with persistent vs. paroxysmal atrial fibrillation: results from the ROCKET-AF Trial. Eur Heart J 2015;36:288-96.

6. Sikorska A, Baran J, Pilichowska-Paszkiet E, et al. Risk of left atrial appendage thrombus in patients scheduled for ablation for atrial fibrillation: beyond the CHA2DS2VASc score. Pol Arch Med Wewn 2015:125:921-8.

7. Gawałko M, Kapłon-Cieślicka A, Budnik M, et al. Comparison of different oral anticoagulant regimens in patients with atrial fibrillation undergoing ablation or cardioversion. Pol Arch Intern Med 2017;127:823-31.

8. Vittinghoff $E$, McCulloch CE. Relaxing the rule of ten events per variable in logistic and Cox regression. Am J Epidemiol 2007;165:710-8.

9. Ntaios $G$, Vemmou A, Koroboki E, et al. The type of atrial fibrillation is associated with long-term outcome in patients with acute ischemic stroke. Int J Cardiol 2013;167:1519-23.

10. Batra G, Svennblad B, Held C, et al. All types of atrial fibrillation in the setting of myocardial infarction are associated with impaired outcome. Heart 2016;102:926-33. 
11. Ganesan AN, Chew DP, Hartshorne T, et al. The impact of atrial fibrillation type on the risk of thromboembolism, mortality, and bleeding: a systematic review and metaanalysis. Eur Heart J 2016;37:1591-602.

12 Boriani G, Botto GL, Padeletti L, et al. Italian AT-500 Registry Investigators. Improving stroke risk stratification using the CHADS2 and CHA2DS2-VASc risk scores in patients with paroxysmal atrial fibrillation by continuous arrhythmia burden monitoring. Stroke 2011;42:1768-70.

13. Chou CC, Lien LM, Chen WH, et al. Adults with late stage 3 chronic kidney disease are at high risk for prevalent silent brain infarction: a population-based study. Stroke 2011:42:2120-5.

14. Piccini JP, Stevens SR, Chang Y, et al. Renal dysfunction as a predictor of stroke and systemic embolism in patients with nonvalvular atrial fibrillation: validation of the R(2)CHADS(2) index in the ROCKET AF (Rivaroxaban Once-daily, oral, direct factor Xa inhibition Compared with vitamin $\mathrm{K}$ antagonism for prevention of stroke and Embolism Trial in Atrial Fibrillation) and ATRIA (AnTicoagulation and Risk factors In Atrial fibrillation) study cohorts. Circulation 2013;127:224-32.

15. Bautista J, Bella A, Chaudhari A, et al. Advanced chronic kidney disease in nonvalvular atrial fibrillation: extending the utility of R2CHADS2 to patients with advanced renal failure. Clin Kidney J 2015;8:226-31.

16. Beyer-Westendorf J, Kreutz R, Posch $F_{1}$ et al. The $\mathrm{CHA}_{2} \mathrm{DS}_{2}$-VASc score strongly correlates with glomerular filtration rate and predicts renal function decline over time in elderly patients with atrial fibrillation and chronic kidney disease. Int J Cardiol 2018;253:71-7.

17. Singer $D E$, Chang $Y$, Borowsky $L H$, et al. A new risk scheme to predict ischemic stroke and other thromboembolism in atrial fibrillation: the ATRIA study stroke risk score. J Am Heart Assoc 2013;2:e000250.

18. Kornej J, Hindricks G, Kosiuk J, et al. Renal dysfunction, stroke risk scores (CHADS2, CHA2DS2-VASC, and R2CHADS2), and the risk of thromboembolic events after catheter ablation of atrial fibrillation: the Leipzig Heart Center AF Ablation Registry. Circ Arrhythm Electrophysiol 2013;6:868-74.

19. Chao TF, Lin YJ, Chang SL, et al. R2CHADS2 score and thromboembolic events after catheter ablation of atrial fibrillation in comparison with the CHA2DS2-VASc score. Can J Cardiol 2014;30:405-12.
20. Abumuaileq RR, Abu-Assi E, López-López A, et al. Comparison between CHA2DS2VASc and the new R2CHADS2 and ATRIA scores at predicting thromboembolic event in non-anticoagulated and anticoagulated patients with non-valvular atrial fibrillation BMC Cardiovasc Disord 2015;15:156

21. O'Brien EC, Holmes DN, Thomas L, et al. Incremental prognostic value of renal function for stroke prediction in atrial fibrillation. Int I Cardiol 2019;274

22. Providência R, Faustino A, Paiva L, et al. Cardioversion safety in patients with nonvalvular atrial fibrillation: which patients can be spared transesophageal echocardiography? Blood Coagul Fibrinolysis 2012;23:597-602.

23. Yumei X, Jun H, Shulin W. GW24-e3109 Correlation of CHADS2 and CHA2DS2-VASC scores with left atrial thrombus in Chinese patients with nonvalvular atrial fibrillation. Heart 2013;99:A186.2-A186.

24. Kim MN, Kim SA, Choi JI, et al. Improvement of predictive value for thromboembolic risk by incorporating left atrial functional parameters in the CHADS2 and CHA2DS2 VASc scores. Int Heart J 2015:56:286-92.

25. Coppens M, Eikelboom JW, Hart RG, et al. The CHA2DS2-VASc score identifies those patients with atrial fibrillation and a CHADS2 score of 1 who are unlikely to benefit from oral anticoagulant therapy. Eur Heart J 2013;34:170-6.

26. The Stroke Prevention in Atrial Fibrillation Investigators Committee on Echocardiography. Transesophageal echocardiographic correlates of thromboembolism in high-risk patients with nonvalvular atrial fibrillation. Ann Intern Med 1998;128:639-47.

27. Stöllberger C, Chnupa P, Kronik G, et al. Transesophageal echocardiography to assess embolic risk in patients with atrial fibrillation. ELAT Study Group. Embolism in Left Atrial Thrombi. Ann Intern Med 1998;128:630-8.

28. Stoddard MF, Singh $P$, Dawn B, et al. Left atrial thrombus predicts transient ischemic attack in patients with atrial fibrillation. Am Heart J 2003;145:676-82.

29. Reddy VY, Sievert H, Halperin J, et al. PROTECT AF Steering Committee and Investigators. Percutaneous left atrial appendage closure vs warfarin for atrial fibrillation: a randomized clinical trial. JAMA 2014;312:1988-98.

30. Gloekler S, Saw J, Koskinas KC, et al. Left atrial appendage closure for prevention of death, stroke, and bleeding in patients with nonvalvular atrial fibrillation. Int J Cardiol 2017:249:234-46. 